\title{
Positionspapier zur Flüchtlingsversorgung
}

\section{Eine Stellungnahme zur psychosozialen Versorgung von Flüchtlingen}

\author{
Carryn Danzinger · Matthäus Fellinger · Waltraud Fellinger-Vols · Georg Psota · Johannes Wancata (D) \\ Alice Wimmer - Thomas Wochele-Thoma
}

Eingegangen: 9. Februar 2018 / Angenommen: 14. Februar 2018 / Online publiziert: 7. März 2018

(C) Der/die Autor(en) 2018. Dieser Artikel ist eine Open-Access-Publikation.

Zusammenfassung Diese Publikation umfasst eine Stellungnahme der Österreichischen Gesellschaft für Psychiatrie, Psychotherapie und Psychosomatik und der Österreichischen Gesellschaft für Sozialpsychiatrie zur psychosozialen Versorgung von Flüchtlingen. Es werden zunächst die Hintergründe aktueller Fluchtbewegungen sowie psychische Erkrankungen von Flüchtlingen dargestellt. Anschließend werden Herausforderungen für die psychosoziale Versorgung von Flüchtlingen thematisiert und entsprechende Empfehlungen beschrieben. Empfehlungen sind möglichst rasche reguläre Unterkünfte, eine Sicherung der Basisbedürfnisse und entsprechende Integrationsmaßnahmen, eine rasche Abwicklung der Asylverfahren und insbesondere auch eine Bedarfsorientierte Versorgung, anhand der sogenannten Interventionspyramide des Inter-Agency Standing Committee der WHO. Außerdem wird empfohlen auch kulturspezifische Aspekte zu berücksichtigen in dem zum Beispiel kultursensitives Wissen in die Aus- und Weiterbildung integriert wird.

\section{Dr. C. Danzinger}

Psychosoziales Zentrum ESRA, Wien, Österreich

Dr. M. Fellinger · Univ.-Prof. Dr. J. Wancata $(\bowtie)$

Klinische Abteilung für Sozialpsychiatrie, Medizinische Universität Wien, Währinger Gürtel 18-20, 1090 Wien, Österreich

johannes.wancata@meduniwien.ac.at

Prim. Dr. W. Fellinger-Vols · Chefarzt Prim. Dr. G. Psota Kuratorium für Psychosoziale Dienste Wien, Wien, Österreich

Dr. A. Wimmer · Dr. T. Wochele-Thoma

Caritas der ERzdiözese Wien, Wien, Österreich
Schlüsselwörter Flüchtlinge - Psychische Belastungen · Trauma · Post-traumatische Belastungsstörung · Versorgung

Position paper about the care of refugees A statement about the psychosocial health care of refugees

Summary This paper is general statement about the psychosocial care of refugees, developed by the of the Austrian Society for Psychiatry, Psychotherapy and Psychosomatics as well as by the Austrian Society for Social Psychiatry. Reasons of recent wave of refugees and the usual mental disorders among refugees are described. The increasing number of refugees, asylum seekers and irregular migrants poses a challenge for mental health services in Europe. As a consequence, several expert recommendations were given. As a first step, basic needs of refugees such as regular housing facilities, sufficient nutrition and safety in everyday life should be met. Fast decisions about the applications for asylum and support of social integration through education and employment are essential for mental health. Provision of mental health care should be needs based as described by the intervention pyramid for mental health and psychosocial support as given by the Inter-Agency Standing Committee of the World Health Organization. In addition, culture-specific aspects must be considered in everyday clinical work and the training of health professionals.

Keywords Refugees · Psychological burden · Trauma · Post-traumatic stress disorder $\cdot$ Health care

\section{Präambel}

Aufbauend auf dem Ärzte-Appell der Österreichischen Gesellschaft für Psychiatrie, Psychotherapie und Psy- 
chosomatik vom 23. Juli 2015, in dem die Forderung eines sicheren, stabilen und menschenwürdigen Umfelds für Flüchtlinge und Asylsuchende erhoben wurde, wird in dem vorliegendem Positionspapier eine allgemeine Stellungnahme zur psychosozialen Versorgung von Flüchtlingen aus aktueller Sicht präsentiert. Auch wenn auf bestehende regionale Unterschiede nicht explizit eingegangen werden kann, wird hierbei der Anspruch auf eine österreichweite gültige Empfehlung gestellt, für deren Umsetzung selbstverständlich individuelle regionale Lösungen erforderlich sind.

In der Bundeshauptstadt und dem nahe gelegenen Erstaufnahmezentrum hat sich die Unterbringungssituation gebessert. Große Quartiere wurden verkleinert, die Qualität der Quartiere wurde verbessert, einige ungeeignete Notquartiere wurden geschlossen. Das Thema der Transition bleibt aber auch hier bestehen: von Massenquartieren zu Privatunterkünften sowie von Jugendeinrichtungen zu Strukturen für Erwachsene. Auch Bildungsangebote haben sich seit der letzten Stellungnahme punktuell erweitert. Es mangelt allerdings österreichweit noch immer an niederschwelligen und gut erreichbaren (Bundesländer) Angeboten.

\section{Hintergrund}

Weltweit sind aktuell ca. 65,6 Mio. Menschen auf der Flucht. Dies entspricht der höchsten Zahl von Flüchtlingen in den letzten 20 Jahren. Verantwortlich für den Anstieg sind laut UNHCR die Konflikte in Syrien, Afghanistan, Burundi, der demokratischen Republik Kongo, Mali, Somalia, Süd-Sudan sowie der Ukraine. Derzeit ist der größte und wichtigste Faktor der bereits erwähnte lange währende bewaffnete Konflikt in Syrien. So stieg die Gesamtzahl der registrierten geflüchteten Syrer von $10.000 \mathrm{im}$ Jahr 2010 auf 5,05 Mio. bis Mai 2017, womit erstmals seit 30 Jahren nicht mehr Afghanistan das Herkunftsland der größten Flüchtlingspopulation war. Der größte Teil (2,99 Mio.) der geflüchteten Syrer wurde bis April 2017 in der Türkei aufgenommen [1].

Der bewaffnete Konflikt in Syrien ist also für die größte Fluchtbewegung unserer Zeit verantwortlich. Während der Großteil dieser Flüchtlinge durch die umliegenden Länder Syriens aufgenommen wurde, kam es im Sommer 2015 zu einem deutlichem Anstieg von Flüchtlingen innerhalb der Europäischen Union, die primär über die Türkei und anschließend Griechenland West- und Mitteleuropa erreichten [2]. In der EU wurden 2015 schließlich insgesamt 1.294.000 Asylanträge gestellt. In Relation zur Einwohnerzahl hatte innerhalb der EU Schweden im Jahr 2015 die meisten Flüchtlinge aufgenommen (15 Flüchtlinge auf 1000 Einwohner). Im globalem Vergleich gesehen, ist allerdings kein Mitgliedsstaat der EU unter jenen zehn Ländern, die weltweit die meisten Flüchtlinge beherbergen [1].
In Österreich wurden im Jahr 2015 insgesamt 88.340 Asylanträge gestellt. Durch die Schließung der Balkanroute und den Flüchtlingsdeal mit der Türkei wurden 2016 die Asylanträge auf insgesamt 42.285 halbiert. In den letzten beiden Jahren waren jeweils $9 \%$ der AsylwerberInnen unbegleitete Minderjährige. $66 \%$ der Erwachsenen, die im Jahr 2016 in Österreich um Asyl ansuchten, waren männlich. Der größte Teil der Flüchtlinge stammte aus Afghanistan (28\%) und Syrien $(21 \%)$ [3, 4]. Die 2015 in sieben Flüchtlingseinrichtungen an 514 geflüchteten Erwachsenen in Wien durchgeführte „Displaced Persons in Austria Survey“ Studie [5] zeigte, dass die Befragten im Schnitt wesentlich besser gebildet und weniger traditionellen Einstellungen verhaftet waren als die Bevölkerung im jeweiligen Heimatland.

\section{Psychische Erkrankungen bei Flüchtlingen}

In zahlreichen Studien konnte gezeigt werden, dass Flüchtlinge und Asylsuchende eine sehr vulnerable Gruppe mit besonderen Vorbelastungen darstellen [6, 7]. Nicht nur die traumatisierenden Erlebnisse im Herkunftsland (z. B. Krieg, Verlust nahestehender Menschen, Folter und Misshandlung), sondern auch die mitunter nicht minder belastenden Erfahrungen während der Flucht (z. B. Lebensgefahr, Gewalt und Diskriminierung) oder im asylgewährenden Land erhöhen das Risiko der Erstmanifestation sowie die längere Dauer vorbestehender psychischer Erkrankungen. So konnte gezeigt werden, dass die Wartezeit auf den Asylbescheid ein Prädiktor für das Auftreten einer Posttraumatischen Belastungsstörung (PTBS) oder Depression und für den Schweregrad dieser Erkrankungen ist [8]. Die fehlende Möglichkeit, über die eigene Zukunft bestimmen zu können, Sprachbarrieren sowie fehlende Beschäftigung stellen ebenso Risikofaktoren dar [9].

Speziell bei Flüchtlingen aus Syrien konnte gezeigt werden, dass viele von ihnen unter Depressionen, pathologischen Trauerreaktionen, Belastungsstörungen, Panikattacken oder anderen Angsterkrankungen leiden [10-15]. Überdies ist bei Flüchtlinge und Asylsuchenden das Suizidrisiko signifikant erhöht $[9,16,17]$. Dies und der Umstand, dass die Prävalenz der Posttraumatischen Belastungsstörungen bei Flüchtlingen um ca. das 10-fache erhöht ist, macht es erforderlich, sich mit der psychiatrischen Versorgung dieser Menschen auseinanderzusetzen [6, 8].

Dabei muss allerdings berücksichtigt werden, dass die Stigmatisierung von Menschen mit psychiatrischen Erkrankungen in muslimisch geprägten Ländern im Allgemeinen weiter verbreitet ist als in vielen eurpäischen Länden $[11,12,18]$. Während in diesen Ländern das expressive Zeigen von Emotionen und Leid häufig sozial akzeptiert ist, ist die Zuschreibung einer psychiatrischen Erkrankung nicht nur für die Betroffenen, sondern auch für deren gesamte Familie stigmatisierend und problematisch. Dies beeinflusst 
wesentlich die Bereitschaft, Hilfe zu suchen oder diese anzunehmen [18].

\section{Herausforderungen für das psychosoziale Hilfe- system und Empfehlungen, die zur psychischen Stabilität beitragen}

\section{Erstaufnahmezentren}

Die Bedingungen in Erstaufnahme- bzw. Verteilerzentren beeinflussen das Ausmaß weiterer Belastungen einer mitunter bereits im Ursprungsland oder auf der Flucht traumatisierten Population. Räumliche Beengtheit ohne Rückzugsmöglichkeit, eingeschränkte Bewegungsfreiheit und vor allem die Ungewissheit über die eigene Zukunft, bewirken Gefühle von Ohnmacht und Ausgeliefertsein.

\section{Empfehlung}

- Betroffene sollen möglichst rasch in reguläre und betreute Unterkünfte weiter geleitet werden. Sie benötigen zeitnah muttersprachliche Informationen über das weitere Geschehen, um so zusätzliche Verunsicherungen und psychische Belastungen zu vermeiden.

- Bei Hinweisen auf psychische Erkrankungen ist es wichtig, die betreffenden Personen innerhalb der Betreuungseinrichtung medizinischem bzw. psychologischem Fachpersonal vorzustellen. Falls nötig, müssen ambulante PsychologInnen bzw. PsychiaterInnen hinzugezogen werden. Bei Bedarf ist eine Behandlung einzuleiten, die in einzelnen Fällen auch eine stationäre Aufnahme im Krankenhaus erfordern kann. Um eine qualitative hochwertige Behandlung zu gewährleisten, müssen DolmetscherInnen herangezogen werden.

\section{Sicherung der Basisbedürfnisse}

Trotz deutlich erhöhter Risiken ist es wichtig, zu betonen, dass nicht jeder Flüchtling eine psychische Erkrankung entwickelt oder gar spezialisierte psychiatrische bzw. psychotherapeutische Behandlung benötigt.

Die Sicherung der Grundbedürfnisse ist eine Voraussetzung, die es ermöglicht, die eigenen Ressourcen zu nutzen und die oft extremen Erlebnisse besser $\mathrm{zu}$ verarbeiten. Aus diesem Grund sind unter anderem adäquates Wohnen, ausreichende Versorgung mit Nahrung und Zugang zum Gesundheitssystem sehr bedeutsam. Sinnloses Warten ohne Aufgaben und ohne Beschäftigung hingegen verschlechtern das psychische Befinden. Falls erforderlich, sollte anfangs niederschwellige sozialarbeiterische Unterstützung und Begleitung angeboten werden.

\section{Empfehlung}

- Integrationsmaßnahmen von Beginn an wie z.B. Deutschkurse, Bildungsangebote und die Möglichkeit den Alltag sinnvoll und selbstverantwortlich zu gestalten tragen zur Fähigkeit Alltagsanforderungen autonom zu bewältigen bei und verringern die Abhängigkeit von fremder Hilfe.

- Ein früher Arbeitsmarktzugang verringert die Abhängigkeit von Sozialleistungen und trägt sowohl zur Entwicklung einer Zukunftsperspektive als auch zur psychischen und sozialen Stabilisierung bei.

\section{Kurze Dauer der Asylverfahren}

Langdauernde Asylverfahren steigern die Unsicherheit die eigene Zukunft betreffend. Damit erhöht sich die Vulnerabilität und die Gefahr der Erstmanifestation bzw. der Verschlechterung einer bereits bestehenden psychischen Erkrankung.

\section{Empfehlung}

- Fremdenrechtliche Verfahren sollten möglichst rasch abgewickelt werden.

\section{Aktivierung der sozialen Netzwerke der Betroffenen}

Die Aktivierung von Ressourcen und Coping-Mechanismen der Betroffenen trägt zur Fähigkeit auch schwierige Alltagsanforderungen autonom $\mathrm{zu}$ bewältigen und damit zur psychischen Stabilisierung bei. Beispielsweise können geeignete Personen mit Migrationshintergrund zu MentorInnen geschult werden. Das macht sie zu ExpertInnen in eigener Sache, wodurch sie die Mitglieder ihrer Gemeinschaft unterstützen können. Weiters können unterschiedliche Aktivitäten zu einem (kulturellen) Austausch zwischen Herkunftsland und Ankunftsland beitragen. Dazu gehören auch gemeinsames Kochen, Feste feiern oder schulische und sportliche Veranstaltungen.

\section{Empfehlung}

- Kompetenzen der Flüchtlinge sollen genützt werden, um derartige Angebote zu entwickeln.

\section{Bedarfsorientierte Versorgung}

Extreme Erfahrungen können bei allen Menschen das Risiko erhöhen, eine Belastungsstörung, eine Depression oder eine andere psychische Erkrankung zu entwickeln. Gespräche mit nahestehenden Menschen, sinnvolle Aufgaben im Alltag, Sicherheit vor weiterem Schaden, Rückzugsmöglichkeiten etc. reduzieren das Krankheitsrisiko. Derart hilfreiche Alltagsbedingungen sind in ähnlicher Weise für Flüchtlinge und Asylsuchende von Relevanz und sollten im Sinne ei- 


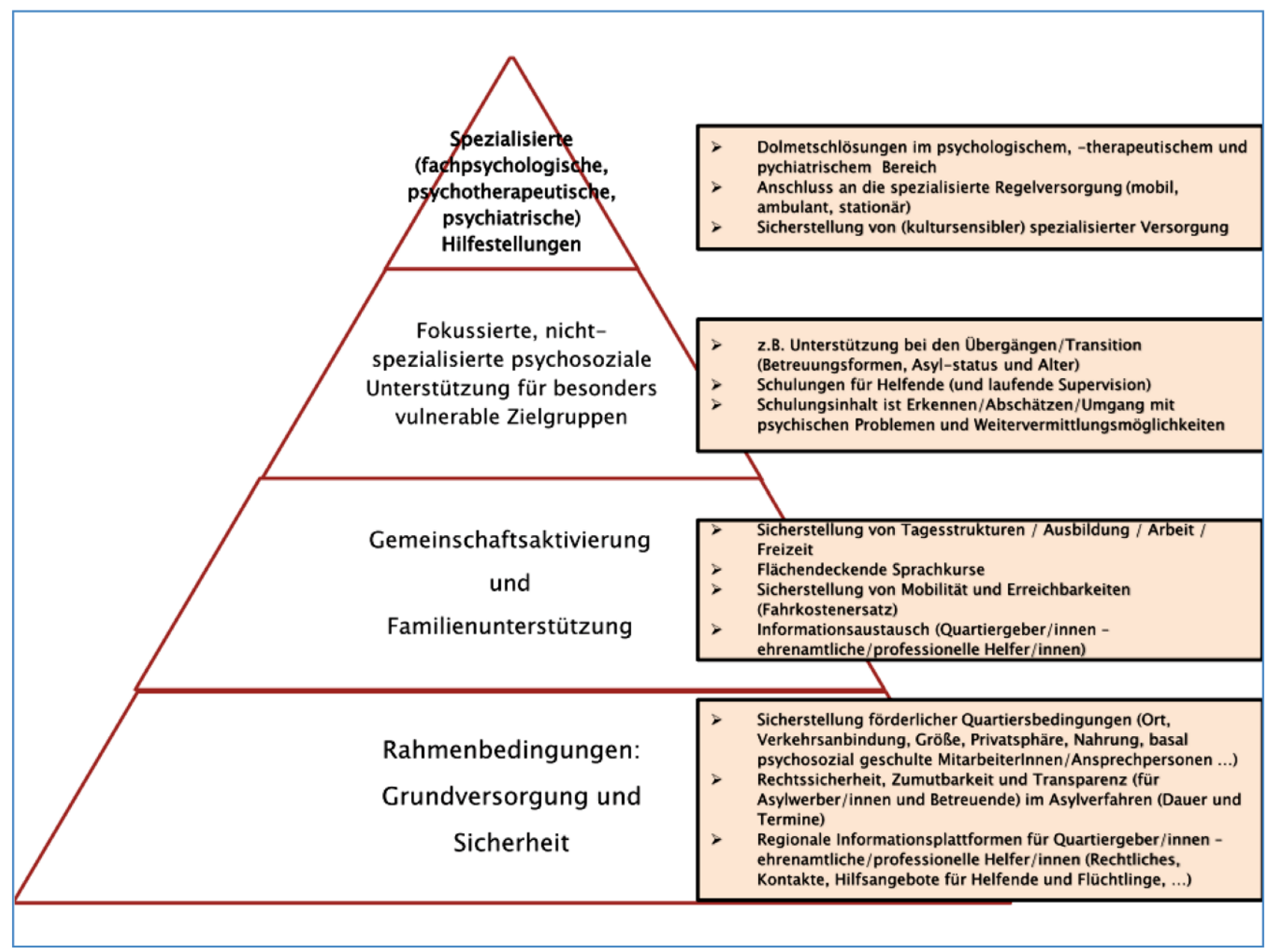

Abb. 1 Interventionspyramide des Inter-Agency Standing Committee (IASC) mit Handlungsebenen [19]. (Übersetzung durch Arbeitsgruppe der Gesundheit Österreich GmbH (Juni 2016))

ner Prävention für alle bestmöglich zur Verfügung stehen.

Bedarforientierte Versorgung bedeutet in diesem Zusammenhang, dass jeder das bekommt, was er oder sie benötigt. Dabei sollen niederschwellige und einfache Angebote Vorrang vor höherschwelligen und aufwendigen Angeboten haben. Wenn allerdings aufwendigere Hilfen benötigt werden, sollten sie entsprechend verfügbar sein. Auf diese Weise kann vermieden werden, dass unnötig Ressourcen verbraucht werden, die anderswo dringend benötigt werden.

Die sogenannte Interventionspyramide des InterAgency Standing Committee [19, 20] verdeutlicht dies auf vier Handlungsebenen, wobei immer zuerst die breite Basis der Pyramide genutzt werden sollte, bevor spezialisierte (in der Abb. 1 weiter oben stehende) und kostenintensive Interventionen zur Anwendung kommen. Damit diese Vorgangsweise funktionieren kann, bedarf es auf jeder Handlungsebene ausreichend qualifizierte Personen.

Personal, das in Kontakt mit Flüchtlingen steht, benötigt kulturelle Sensitivität, kulturspezifisches Wissen und Kompetenz im Erkennen von möglichen Hin- weisen auf psychische Erkrankungen. Das erhöht die Sicherheit der Einschätzung von Auffälligkeiten und reduziert Fehlzuweisungen in spezialsiertere und teurere Angebote. Dazu ist es erforderlich, Personal in Betreuungseinrichtungen, Berater in Anlaufstellen, medizinisches Personal und Beamte zu schulen.

Bei Verdacht auf psychische Erkrankungen sollten fachspezifisch geschulte Personen z. B. PsychologInnen, PsychotherapeutInnen, Pflegepersonen beigezogen werden. Hierzu eignen sich mobile Einheiten oder spezialisiertes Personal in Beratungsstellen. Ziel ist es, abzuklären, ob eine weiterführende psychiatrische-psychotherapeutische Behandlung notwendig ist oder eine niederschwelligere Versorgung vor Ort ausreicht.

Falls erforderlich, werden die Betroffenen nach dieser fachlichen Begutachtung an entsprechende Stellen des Versorgungssystems weitergeleitet.

Wartezeiten für ambulante psychotherapeutische und psychiatrische Behandlungen sind in vielen Regionen Österreichs derzeit oft extrem lang. Das erhöht das Risiko für Symptomverschlechterung und Chronifizierung von Erkrankungen. 


\section{Empfehlung}

- MitarbeiterInnen von Betreuungseinrichtungen sollten basale Informationen über Möglichkeiten fachlicher Unterstützung erhalten.

- Entsprechende Ressourcen müssen im fachspezifischen Regelversorgungssystem auf allen Ebenen zur Verfügung gestellt werden.

\section{Kulturspezifische Aspekte}

Der kulturelle Hintergrund der Flüchtlinge beeinflusst die Präsentation und Attribuierung von psychischen Beschwerden und das Krankheitsverständnis wesentlich. Dadurch ergeben sich Behandlungserwartungen, die die Akzeptanz der hierorts angebotenen Therapien beeinflussen. Daher sind Sprach- und Kulturvermittlung durch professionelle Dolmetscher eine wesentliche Basis für die Kommunikation zwischen Gesundheitsberufen und PatientInnen. Dies ist eine wichtige Grundlage, um ein gemeinsames Krankheitsund Behandlungsverständnis zu erarbeiten und damit eine funktionierende Arzt-Patientenbeziehung zu etablieren. Zusätzlich können niederschwellige Informationen in den Muttersprachen bzw. Pictogramme zu Krankheitsbildern und Angeboten auf allen Ebenen hilfreich sein.

\section{Empfehlung}

- Sprach- und Kulturvermittlung durch professionelle DolmetscherInnen sind in ausreichendem Umfang sicherzustellen.

- Es ist sicher zu stellen, dass kultursensitves Wissen in die Aus- und Weiterbildung integriert wird.

Anmerkung: Obwohl von großer Bedeutung geht das vorliegende Positionspapier nicht auf die Dokumentation von Folteropfern und die speziellen Bedürfnisse von Kindern und Jugendlichen unter den Flüchtlingen ein. Diesbezüglich finden sich detaillierte Informationen an anderer Stelle [21-23].

Funding Open access funding provided by Medical University of Vienna.

Interessenkonflikt Alle Autoren haben in ihrer täglichen Arbeit auch mit Flüchtlingen und Asylsuchenden zu tun. Darüber hinaus geben C. Danzinger, M. Fellinger, W. FellingerVols, G. Psota, J. Wancata, A. Wimmer und T. Wochele-Thoma an, dass kein Interessenkonflikt besteht.

Open Access Dieser Artikel wird unter der Creative Commons Namensnennung 4.0 International Lizenz (http:// creativecommons.org/licenses/by/4.0/deed.de) veröffentlicht, welche die Nutzung, Vervielfältigung, Bearbeitung, Verbreitung und Wiedergabe in jeglichem Medium und Format erlaubt, sofern Sie den/die ursprünglichen Autor(en) und die Quelle ordnungsgemäß nennen, einen Link zur Creative Commons Lizenz beifügen und angeben, ob Änderungen vorgenommen wurden.

\section{Literatur}

1. UNHCR. World at war-global trends-forced displacement in 2014. 2017. http://www.unhcr.at/service/zahlenund-statistiken.html.Zugegriffen: 10.12.2017

2. Abbasi K, PatelK, GodleeF.Europe's refugee crisis: an urgent call for moral leadership. BMJ.2015;351:h4833.

3. Bundesministerium für Inneres. Asylstatistik 2015. 2015. http://www.bmi.gv.at/301/Statistiken/files/ Jahresstatistiken/Asyl_Jahresstatistik_2015.pdf. Zugegriffen: 10.12.2017

4. Bundesministerium für Inneres. Asylstatistik 2016. 2016. http://www.bmi.gv.at/301/Statistiken/files/ Jahresstatistiken/Jahresstatistik_Asyl_2016.pdf. Zugegriffen: 10.12 .2017

5. Buber-Ennser I, Kohlenberger J, Rengs B, AlZalakZ, Goujon A, Striessnig E, Potancokova M, Gisser R, Testa MR, Lutz W. Human capital, values, and attitudes of persons seeking refuge in Austria in 2015. PLoS ONE. 2016;11(9):e163481.

6. Fazel M, Wheeler J, Danesh J. Prevalence of serious mental disorder in 7000 refugees resettled in western countries: a systematic review. Lancet. 2005;365(9467):1309-14.

7. Porter M, Haslam N. Predisplacement and postdisplacement factors associated with mental health of refugees and internally displaced persons: a meta-analysis. JAMA. 2005;294:602-12.

8. Knipscheer JW, Sleijpen M, Mooren T, Ter HFJ, van der Aa N. Trauma exposure and refugee status as predictors of mental health outcomes in treatment-seeking refugees. BJPsych Bull.2015;39:178-82.

9. Aoe T, Shetty S, Sivilli T, Blanton C, Ellis H, Geltman PL, et al. Suicidal ideation and mental health of Bhutanese refugees in the United States.JImmigr Minor Health. 2016;18:828-35.

10. AcarturkC,KonukE, CetinkayaM,SenayI,SijbrandijM,Cuijpers P, AkerT. EMDR for Syrian refugees with posttraumatic stress disorder symptoms: results of a pilot randomized controlled trial. Eur J Psychotraumatol. 2015;6:27414.

11. de Jong JT, Komproe IH, Van Ommeren M. Common mental disorders in postconflict settings. Lancet. 2003;361(9375):2128-30.

12. Hassan G, Ventevogel P, Jefee-Bahloul H, Barkil-Oteo A, Kirmayer LJ. Mental health and psychosocial wellbeing of Syrians affected by armed conflict. Epidemiol Psychiatr Sci. 2016;25:129-41.

13. Momartin S, Silove D, Manicavasagar V, Steel Z. Complicated grief in Bosnian refugees: associations with posttraumatic stress disorder and depression. Compr Psychiatry. 2004;45:475-82.

14. Shrestha NM, Sharma B, Van Ommeren M, RegmiS, Makaju $\mathrm{R}$, Komproe I, et al. Impact of torture on refugees displaced within the developing world: symptomatology among Bhutanese refugees in Nepal. JAMA. 1998;280:443-8.

15. Steel Z, Chey T, Silove D, Marnane C, Bryant RA, van Ommeren $M$. Association of torture and other potentially traumatic events with mental health outcomes among populations exposed to mass conflict and displacement: a systematic review and meta-analysis. JAMA. 2009;302:537-49.

16. Hagaman AK, Sivilli TI, Ao T, Blanton C, Ellis H, Lopes Cardozo B, Shetty S. An investigation into suicides among Bhutanese refugees resettled in the United States between 2008 and 2011. J Immigr Minor Health. 2016;18:819-27.

17. Rahman A, Hafeez A. Suicidal feelings run high among mothers in refugee camps: a cross-sectional survey. Acta Psychiatr Scand. 2003;108:392-3.

18. Ciftici A, Jones N, Corrigan W. Mental health stigma in the muslim community. J Muslim Ment Health. 2013;7:17-32. 


\section{übersicht}

19. Inter-Agency Standing Committee (IASC). IASC guidelines on mental health and psychosocial support in emergency settings. Geneva: IASC; 2007. http://www. who.int/mental_health/emergencies/guidelines_iasc_ mental_health_psychosocial_june_2007.pdf.

20. Inter-Agency Standing Committee (IASC). Reference group for mental health and psychosocial support in emergency settings. Mental health and psychosocial support in humanitarian emergencies: what should humanitarian health actors know? Geneva: IASC; 2010.

21. Anagnostopoulos DC, Heberbrand J, Eliez S, Doyle MB, Klasen H, Crommen S, Cuhadaroğlu FC, Pejovic-Milovancevic M, Herreros O, Minderaa R, Karwautz A, Svedin CG, Raynaud JP. European Society of Child and Adolescent
Psychiatry: Position statement on mental health of child and adolescent refugees. European ChildAdolescentPsych. 2016;25(7):673-676

22. Hebebrand J, Anagnostopoulos D, Eliez S, Linse H, PejovicMilovancevic M, Klasen H A first assessment of the needs of young refugees arriving in Europe: What mental health professionals need to know. European Child Adolescent Psych. 2016;25(1):1-6

23. Office of the UN High Commissioner for Human Rights (2004): Istanbul Protocol. Manual on the Effective Investigation and Documentation of Torture and Other Cruel, Inhuman or Degrading Treatment or Punishment. Professional Training Series 8(1). Online unter: http://www. unhchr.ch/pdf/8istprot.pdf 